\title{
Cancer patients taking herbal medicines: a review of clinical purposes, associated factors, and perceptions of benefit or harm
}

Article

Accepted Version

Poonthananiwatkul, B., Howard, R. L., Williamson, E. M. and Lim, R. H. M. (2015) Cancer patients taking herbal medicines:

a review of clinical purposes, associated factors, and perceptions of benefit or harm. Journal of Ethnopharmacology, 175. pp. 58-66. ISSN 0378-8741 doi:

https://doi.org/10.1016/j.jep.2015.08.052 Available at https://centaur.reading.ac.uk/42755/

It is advisable to refer to the publisher's version if you intend to cite from the work. See Guidance on citing.

To link to this article DOI: http://dx.doi.org/10.1016/j.jep.2015.08.052

Publisher: Elsevier

All outputs in CentAUR are protected by Intellectual Property Rights law, including copyright law. Copyright and IPR is retained by the creators or other copyright holders. Terms and conditions for use of this material are defined in the End User Agreement. 


\section{CentAUR}

Central Archive at the University of Reading

Reading's research outputs online 


\section{Author's Accepted Manuscript}

Cancer patients taking herbal medicines: A review of clinical purposes, associated factors, and perceptions of benefit or harm

Benjawan Poonthananiwatkul, Rachel L Howard, Elizabeth M Williamson, Rosemary H M Lim

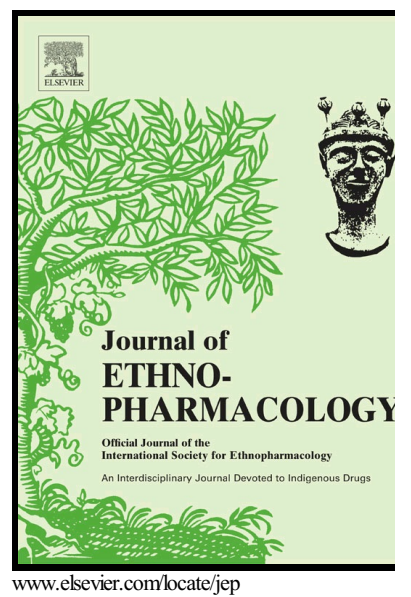

PII: $\quad$ S0378-8741(15)30119-7

DOI: $\quad$ http://dx.doi.org/10.1016/j.jep.2015.08.052

Reference: JEP9717

To appear in: Journal of Ethnopharmacology

Received date: 1 June 2015

Revised date: 18 August 2015

Accepted date: 30 August 2015

Cite this article as: Benjawan Poonthananiwatkul, Rachel L Howard, Elizabeth $\mathrm{N}$ Williamson and Rosemary H M Lim, Cancer patients taking herbal medicines: f review of clinical purposes, associated factors, and perceptions of benefit o $\mathrm{h}$ a $\mathrm{r} \quad \mathrm{m}$, Journal of Ethnopharmacology http://dx.doi.org/10.1016/j.jep.2015.08.052

This is a PDF file of an unedited manuscript that has been accepted fo publication. As a service to our customers we are providing this early version o the manuscript. The manuscript will undergo copyediting, typesetting, an review of the resulting galley proof before it is published in its final citable form Please note that during the production process errors may be discovered whic could affect the content, and all legal disclaimers that apply to the journal pertain 
Abstract

Ethnopharmacological relevance: Cancer patients in all cultures are high consumers of herbal medicines (HMs) usually as part of a regime consisting of several complementary and alternative medicine (CAM) modalities, but the type of patient, the reasons for choosing HM-CAM regimes, and the benefits they perceive from taking them are poorly understood. There are also concerns that local information may be ignored due to language issues. This study investigates aspects of HM-CAM use in cancer patients using two different abstracting sources: Medline, which contains only peer-reviewed studies from SCI journals, and in order to explore whether further data may be available regionally, the Thai national databases of HM and CAM were searched as an example.

Materials and Methods: the international and Thai language databases were searched separately to identify relevant studies, using key words chosen to include HM use in all traditions. Analysis of these was undertaken to identify socio-demographic and clinical factors, as well as sources of information, which may inform the decision to use HMs.

Results: Medline yielded 5,638 records, with 49 papers fitting the criteria for review. The Thai databases yielded 155, with none relevant for review. Factors associated with HMCAM usage were: a younger age, higher education or economic status, multiple chemotherapy treatment, late stage of disease. The most common purposes for using HMCAM cited by patients were to improve physical symptoms, support emotional health, stimulate the immune system, improve quality of life, and relieve side-effects of conventional treatment.

Conclusions: Several indicators were identified for cancer patients who are most likely to take HM-CAM. However, interpreting the clinical reasons why patients decide to use HMCAM is hampered by a lack of standard terminology and thematic coding, because patients' own descriptions are too variable and overlapping for meaningful comparison. Nevertheless, fears that the results of local studies published regionally are being missed, at least in the case of Thailand, appeared to be unfounded.

Cancer patients taking herbal medicines: a review of clinical purposes, associated factors, and perceptions of benefit or harm

Benjawan Poonthananiwatkul, Rachel L Howard, Elizabeth M Williamson*, Rosemary H M Lim. University of Reading School of Pharmacy, Whiteknights, Reading RG6 6AP *Author for correspondence. 
Abstract

Ethnopharmacological relevance: Cancer patients in all cultures are high consumers of herbal medicines (HMs) usually as part of a regime consisting of several complementary and alternative medicine (CAM) modalities, but the type of patient, the reasons for choosing such HM-CAM regimes, and the benefits they perceive from taking them are poorly understood. There are also concerns that local information may be ignored due to language issues. This study investigates aspects of HM-CAM use in cancer patients using two different abstracting sources: Medline, which contains only peer-reviewed studies from SCI journals, and in order to explore whether further data may be available regionally, the Thai national databases of HM and CAM were searched as an example.

Materials and Methods: the international and Thai language databases were searched separately to identify relevant studies, using key words chosen to include HM use in all traditions. Analysis of these was undertaken to identify socio-demographic and clinical factors, as well as sources of information, which may inform the decision to use HMs.

Results: Medline yielded 5,638 records, with 49 papers fitting the criteria for review. The Thai databases yielded 155, with none relevant for review. Factors associated with HMCAM usage were: a younger age, higher education or economic status, multiple chemotherapy treatment, late stage of disease. The most common purposes for using HMCAM cited by patients were to improve physical symptoms, support emotional health, stimulate the immune system, improve quality of life, and relieve side-effects of conventional treatment.

Conclusions: Several indicators were identified for cancer patients who are most likely to take HM-CAM. However, interpreting the clinical reasons why patients decide to use HMCAM is hampered by a lack of standard terminology and thematic coding, because patients' own descriptions are too variable and overlapping for meaningful comparison. Nevertheless, fears that the results of local studies published regionally are being missed, at least in the case of Thailand, appeared to be unfounded.

Keywords: cancer patient, complementary and alternative medicine, herbal medicine, food supplement, review.

Introduction

Cancer patients in all parts of the world are high users of herbal medicines (HMs), which they choose for clinical reasons related to their cancer diagnosis (Poonthananiwatkul 2015) 
and which they usually take as part of a regime consisting of several complementary and alternative medicine (CAM) modalities (Alsanad et al 2014; McLay et al 2012). The contribution of HMs cannot easily be separated from those of other forms of CAM, although they are more likely to possess pharmacological effects and/or interact with conventional medicines. The specific reasons why patients take HM-CAM regimes have not been completely identified but include trying to actively treat cancer, reduce symptoms of the disease, ameliorate side effects associated with conventional treatments, prevent further recurrence or metastasis of the cancer, and to enhance general health in order to deal with the disease and its treatment (Poonthananiwatkul 2015; Alsanad et al 2014; Ernst 2009). A recent study of cancer patients at a traditional medicine hospice in Thailand suggested that in general herbal medicines were perceived to provide more benefit than harm, and a preliminary assessment of the herbal regime, using changes in symptom burden after staying at the hospice, supported this (Poonthananiwatkul 2015). HMs can be registered as medicines in the European Union, but not in most other countries, and 'nutritional' products are poorly regulated everywhere. HMs are often sold as 'food' or 'dietary' supplements to circumvent the regulations; however, as they are taken for therapeutic purposes they are considered to be HMs for the purposes of this study. The first step to addressing the problem of uncontrolled use of HMs as self-medication is therefore to explore the reasons why patients feel the need to take them. Perceptions of the efficacy and safety of these medicines influence the products chosen, although patients are unlikely to consider the indirect consequences of taking these medicines, including their interaction with conventional medicines or other supplements (Goey et al 2014; Zeller et al 2013). The issues posed by combining herbal medicines with conventional drugs have been well documented over the last decade (e.g. Alsanad et al 2014; McLay et al 2012; Williamson et al 2013) and patients in many countries are now being advised to avoid taking herbal medicines during conventional cancer treatment, although no published evidence is available to confirm this as a policy. The objective of this review is to summarize the socio-demographic and other factors that influence HM-CAM use in cancer patients, and their perceptions towards their benefit or harm.

Materials and methods

Search strategy

Data collected in ethnobotanical research has well-documented weaknesses, as critically reviewed by Heinrich et al (2009), and one of these is that datasets compiled regionally in local languages may not be available internationally. In order to investigate whether any 
such 'hidden studies' were available, two separate reviews were carried out: the first, a search of Science Citation Indexed, peer-reviewed journals in Medline; the second, a search of the national databases in Thailand. The purpose of the Thai review was to act as an example to investigate whether extra information could be gained by casting the net more widely, despite the unreliability of non-peer-reviewed sources. The Thai databases were used as a test case because Thailand is a very high user of herbal medicines, they are comprehensive and we had access to the full dataset. The searches were restricted to 2003 onwards to provide a contemporary context and also because as a preliminary search found very few relevant studies prior to this. Even peer-review cannot guarantee quality so as many details as possible about each study (method, sample size, other findings) are included in table 1 to add context.

Information sources and searches

The global database Medline and the Thai on-line databases [Thailand Library Integrated System (ThaiLIS), Library of National Research Council of Thailand, Health Systems Research Institute Library, Thai Theses Online, Institute of Thai Traditional Medicine, Journal of Thai Traditional and Alternative medicine] (Thai Government 2014) were searched to identify literature on the experiences, attitudes or perceptions of cancer patients who had taken herbal medicines, using the following terms or their Thai language versions:

1) Complementary

2) Alternative

3) Medicine

4) Herbs

5) 1 or 2 or 3 or 4

6) Cancer

7) Attitude

8) 5 and 6 and 7

9) Limited to English

10) Limited to 2003 and 2014

The off-line Khampramong research database was also searched using the same terms, as an example of an institutional data resources. All English language studies published between 2003 and 2014 identifying the experiences/attitudes/ perceptions/ intended purposes of cancer patients regarding HM were included. Review articles, operational (e.g. clinical guidelines) and health services (e.g. cancer screening) research, case reports, 
studies on CAM which did not include HM use or surveys of other parties (such as physicians and other healthcare providers), and laboratory and animal studies were excluded. Studies looking purely at prevalence, trends and costs of herbal medicines were also omitted, as were studies on herb-drug combinations or side effects.

Data extraction

Full papers were obtained for studies considered relevant (figs 1 and 2) and read through by BP. To ensure validity, they were checked by Dr Saud Alsanad (College of Medicine, Al-Imam Mohammad Bin Saud Islamic University, Riyadh, KSA). The following data were extracted from the selected papers: author, year of publication, country, method used, response rate (\%), sample size or calculation reported, cancer type, factors related to use of herbal medicine, purposes and thematic concepts cited for use. Factors such as age, gender, education level, income, type of cancer, previous conventional treatment and HM use were recorded, and also sources of information, which may influence decisions to use these products. Perceptions of benefit or harm resulting from taking these products were evaluated, but it must be emphasised that these are the opinions of patients who voluntarily took part in the studies cited, and are reported without any corroboration by independent assessment, clinical examination or biochemical tests. This is an intrinsic but unavoidable weakness of such studies.

5,638 records were found in the Medline database, but only 170 were judged relevant based on the title, i.e. they specifically examined herbal medicine use in cancer patients. 49 papers were eventually included in the review, as shown in figure 1. The Thai database search initially found 155 records, and 14 studies of herbal medicine use in cancer patients were selected based on the title (Fig 2). Titles and abstracts were read through by author BP and validated by a Thai speaker, Dr Supaporn Bunsiriluck (Sirindhorn College of Public Health, Thailand). No Thai language studies were found which fulfilled the criteria for inclusion, so no further analysis was undertaken for these studies.

\section{Insert figures 1 and 2 here}

Figure 1: Flow chart of the study selection process from the Medline database

Citations identified from databases $(n=5,638)$

Studies not relevant based on the title of the

(These were review articles, operational rese not involving herbal medicines, those concer 
Potentially relevant articles regarding herbal medicine use by cancer patients from databases ( $\mathrm{n}=$ Studies not relevant and excluded based on $\mathrm{t}$

(Excluded papers focussed on the prevalence of herbal medicines.)

Records eligible for full text screening, i.e. those regarding overall issues involved in using herb experiences/attitudes/perceptions by cancer patients $(n=60)$

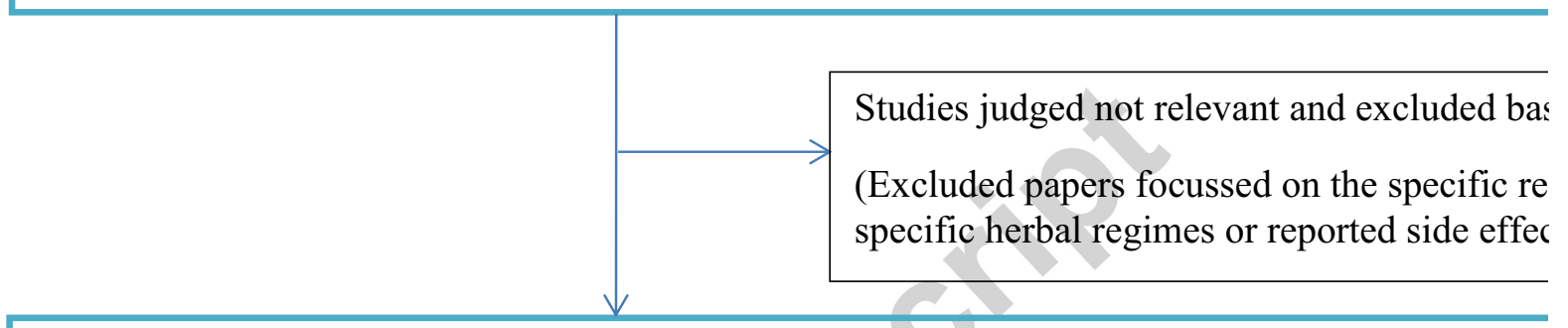

Included studies on experiences/ attitude/ perceptions of cancer patients after using herbs $(\mathrm{n}=49)$

Figure 2: Flow chart of the study selection process from the Thai national databases

Citations identified from all Thai databases $(n=155)$

\begin{tabular}{|l|l|l}
\hline Potentially relevant articles of CAM and herbal medicine use in cancer patients ( $\mathrm{n}=14)$ \\
\hline \begin{tabular}{|l|l|} 
No records eligible for full text screening concerning herbal medicine or CAM (experiences/attit \\
perceptions of cancer patients) (n=0)
\end{tabular} \\
\begin{tabular}{|l|l} 
Studies not relevant based on the review of ; \\
laboratory studies, animal studies, those invc \\
(Excluded papers focussed mainly on the pa \\
herbal medicine)
\end{tabular} \\
\hline
\end{tabular}

Results 
Purposes cited by cancer patients for taking herbal medicines as part of a CAM regime The main reasons given by cancer patients as to why they use HM-CAM are illustrated in fig 3; which in fact shows that this type of analysis is not particularly useful, as there is so much overlap in potential meaning in the reasons cited by the patients themselves. We used the terms cited in the studies to try to avoid misinterpretation, but these are highly subjective. Not all studies used the same parameters, terminologies and methodologies, and more than one purpose was frequently cited by patients who also often used multiple types of CAM. Although all the studies reviewed included HM as a category of CAM, most could not differentiate findings from each modality, so it is not possible to ascribe all the findings to HM use. A further complication is that the line between HM and dietary supplements is not clear, and can depend on non-clinical issues such as legal classification.

CAM and HM in particular are to alleviate physical symptoms associated with cancer, but this category could easily include 'improving general health and the ability to fight the disease', as well as 'treating cancer' and 'improving quality of life', although these reasons were also described specifically. Similarly, 'supporting emotional or mental health' could include 'taking an active role in treatment', 'managing stress' and 'feeling in control'. 'Stimulating or boosting the immune system' was also considered very important, and whereas few studies reported that using HM was intended to achieve a longer life-span, this is implicit in most other categories such as preventing recurrence and treating or curing cancer. Only one study one suggested that 'dissatisfaction with conventional medicine', but the fact that so many cancer patients use HM-CAM suggests that they do not think that conventional medicine has all the answers. Fig 3 therefore also illustrates the importance of using standardised terminology or the use of thematic coding, rather than relying on patients' own descriptions verbatim for this kind of study. Despite this, almost all of the categories relate to the desire to be actively involved in treatment, and the impetus to use HM-CAM comes from patients, rather than practitioners.

Figure 3 Illustration of purposes cited by cancer patients for using herbal medicines as part of a CAM regime 


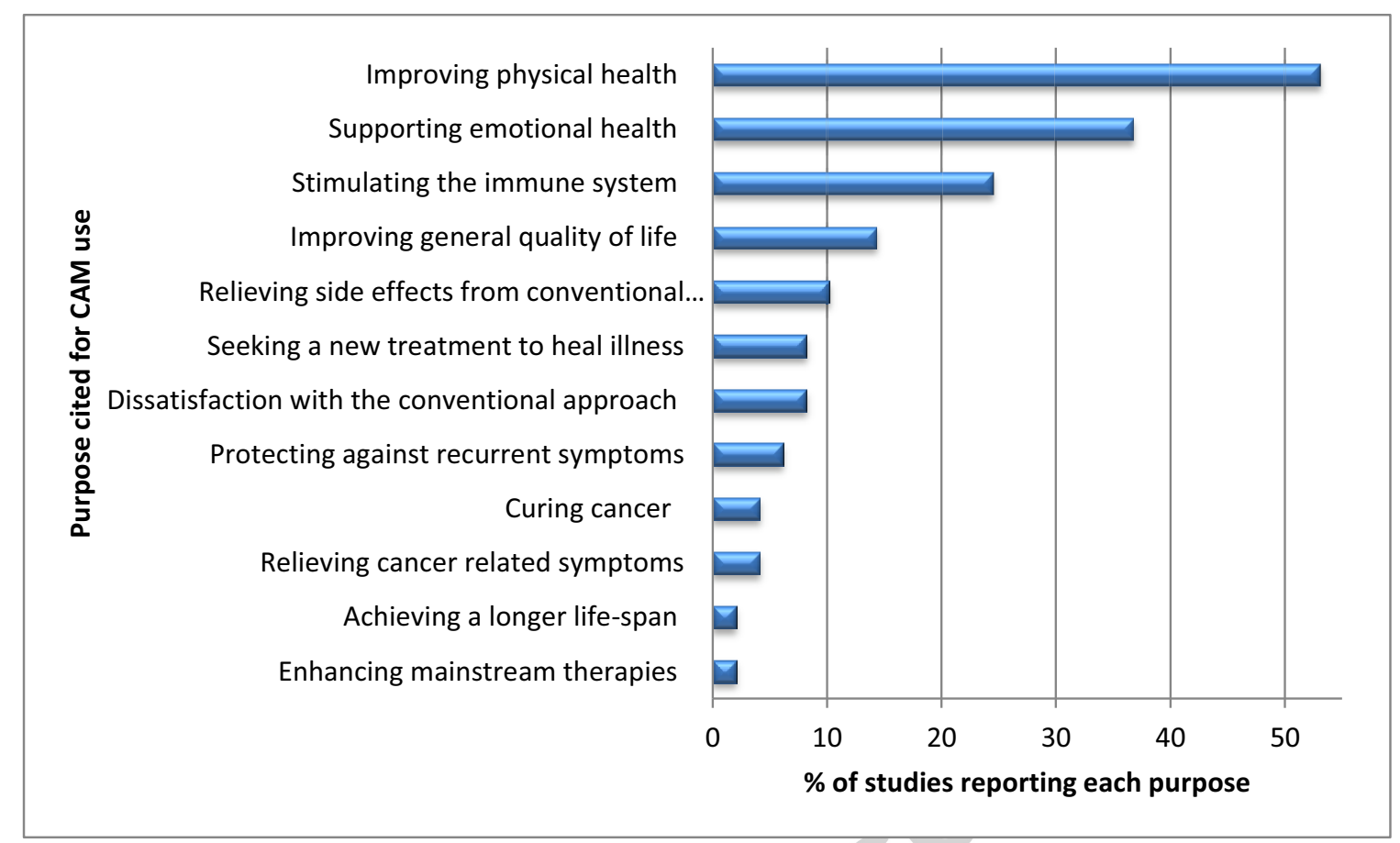




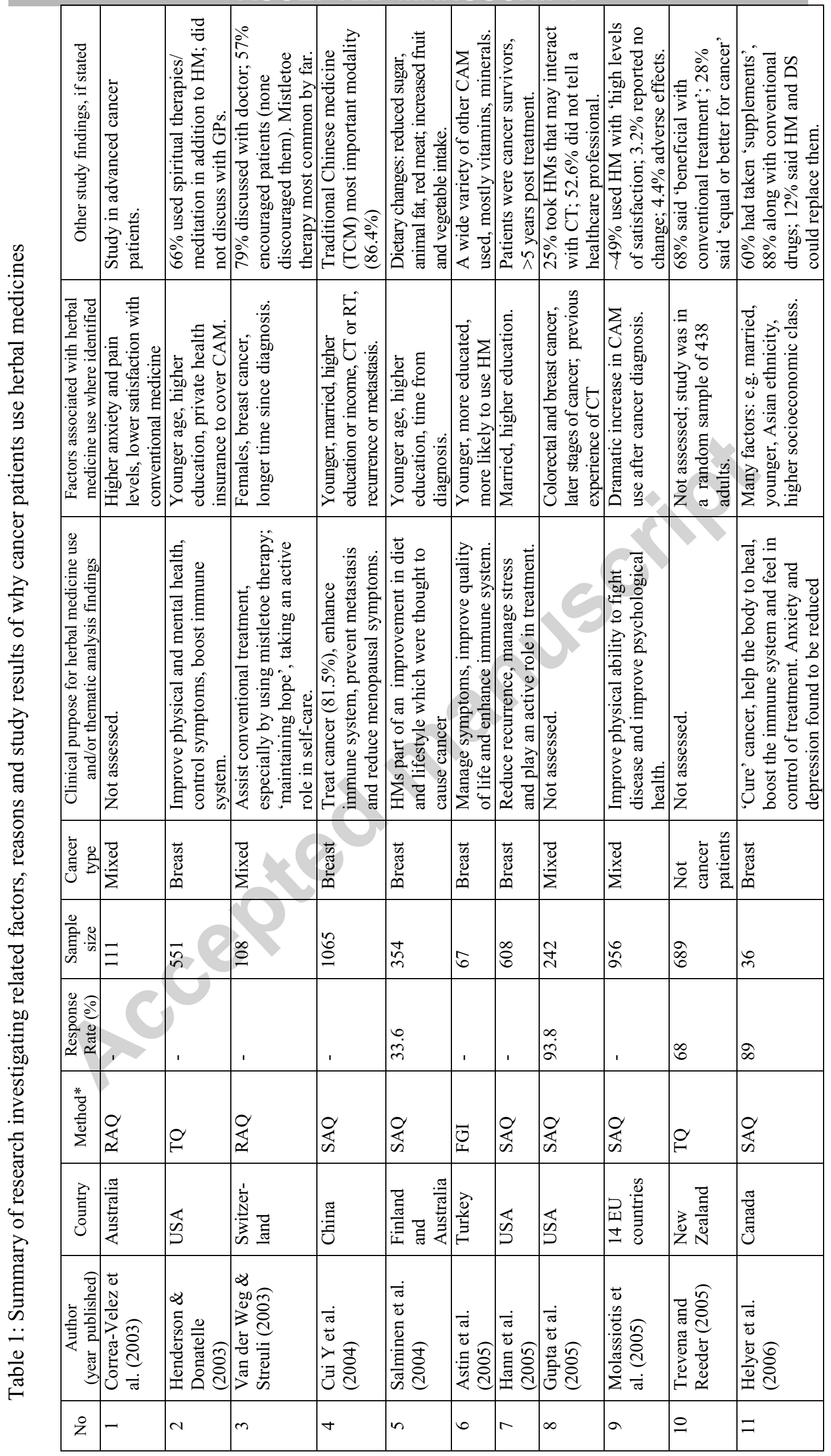




\begin{tabular}{|c|c|c|c|c|c|c|c|c|c|c|c|c|c|}
\hline & 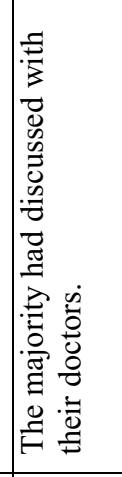 & 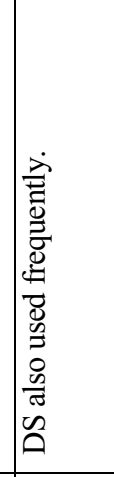 & 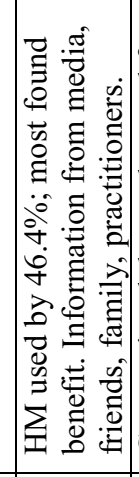 & 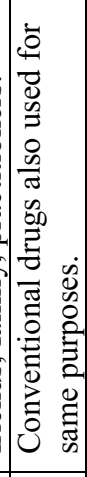 & 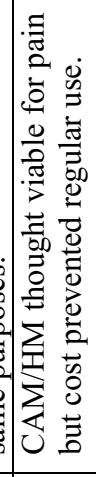 & 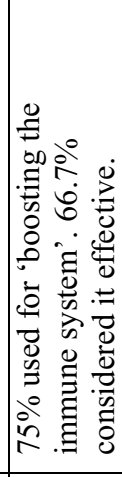 & 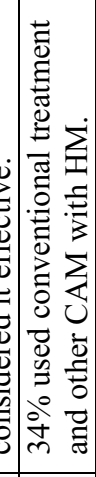 & 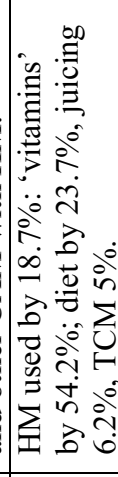 & 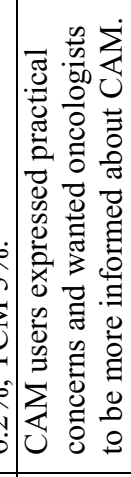 & 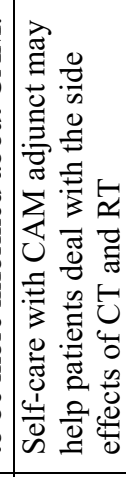 & 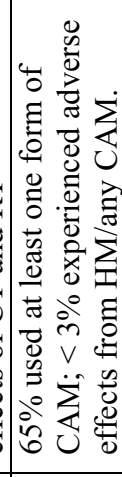 & 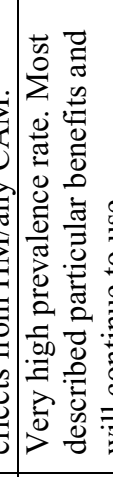 & 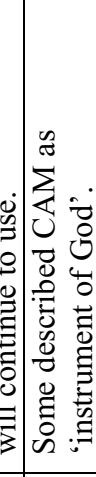 \\
\hline & 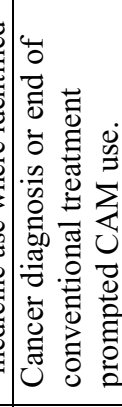 & 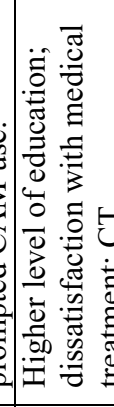 & 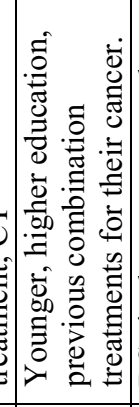 & 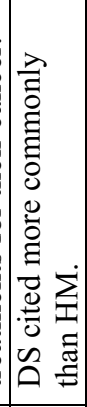 & 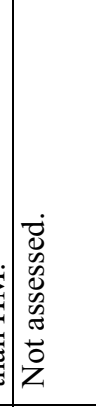 & 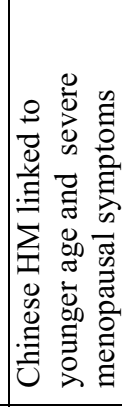 & 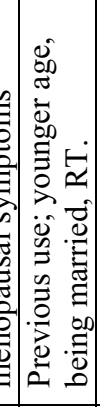 & 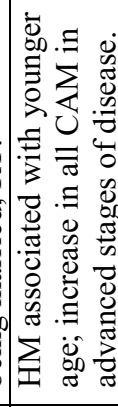 & 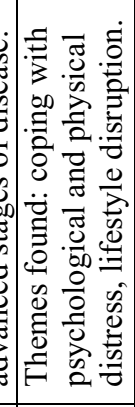 & 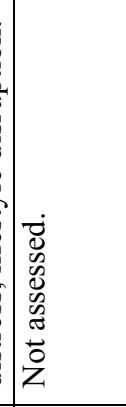 & 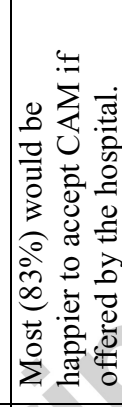 & 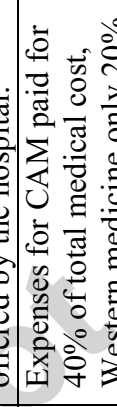 & 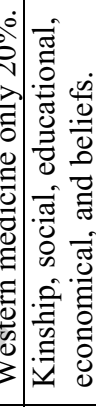 \\
\hline & 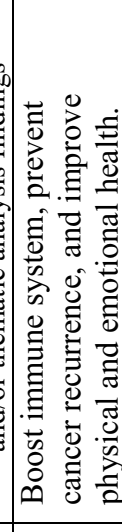 & 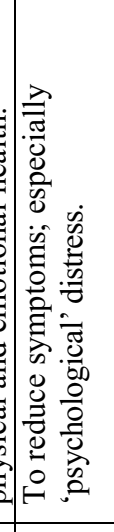 & 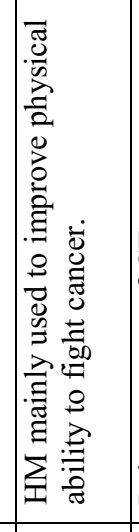 & 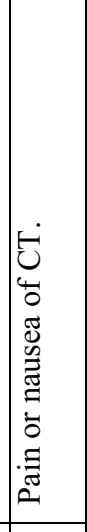 & 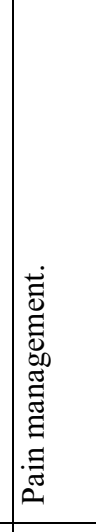 & 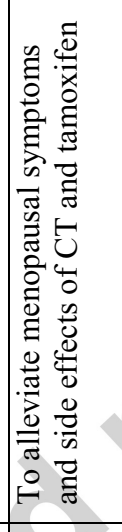 & 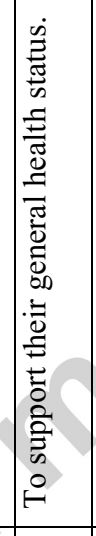 & 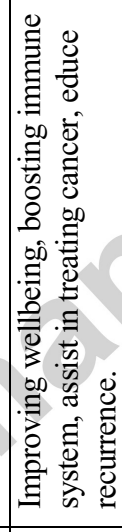 & 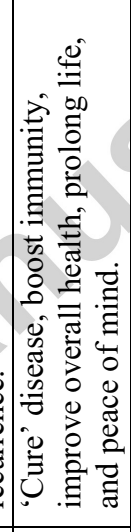 & 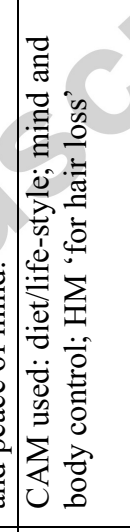 & 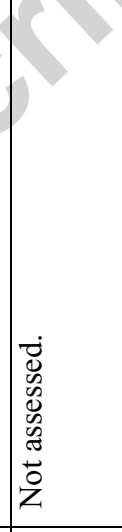 & 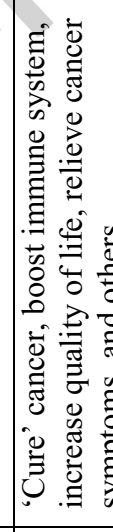 & 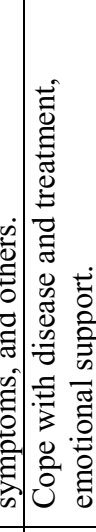 \\
\hline & $\stackrel{\square}{*}$ & 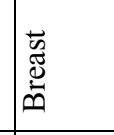 & 㤎 & $\bar{\Phi}$ & 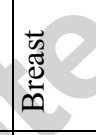 & 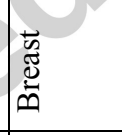 & 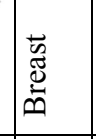 & 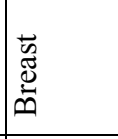 & $\begin{array}{l}\overrightarrow{\widetilde{U}} \\
\stackrel{\times}{\Sigma}\end{array}$ & 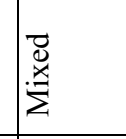 & 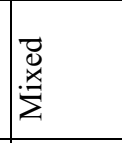 & : & 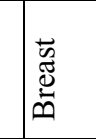 \\
\hline & 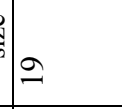 & $\stackrel{2}{2}$ & & m & i & 咅 & సิ & | & r & & & 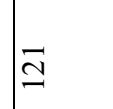 & \\
\hline & & & & & & & & & & & ثن & & \\
\hline & $\vec{\Theta}$ & $\frac{9}{2}$ & $\mid \begin{array}{l}0 \\
\mathbb{x} \\
n\end{array}$ & 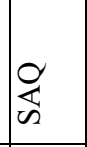 & $\overrightarrow{\overline{\tilde{\omega}}}$ & $\alpha$ & $\frac{a}{d}$ & $\frac{a}{a_{x}}$ & $\bar{\Xi}$ & & $\begin{array}{l}0 \\
\text { d } \\
\text { in }\end{array}$ & $\sqrt{2}$ & $\overline{\tilde{n}}$ \\
\hline 郎 & 吾 & 岁 & 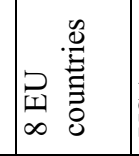 & $\begin{array}{l}\varangle \\
\tilde{s}\end{array}$ & $\begin{array}{l}\mathbb{x} \\
2 \\
2\end{array}$ & .ः & $\mid \frac{\tilde{E}}{3}$ & 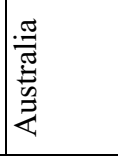 & 言 & T & 嵒 & 造 & $\begin{array}{l}\mathbb{x} \\
\tilde{s}\end{array}$ \\
\hline 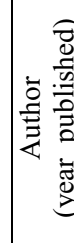 & 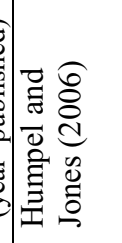 & 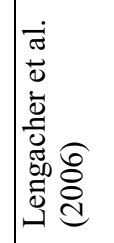 & 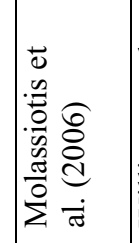 & 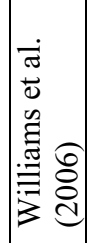 & 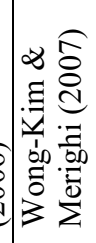 & 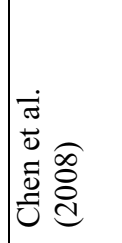 & 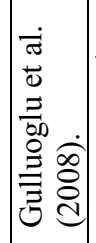 & 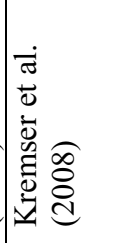 & 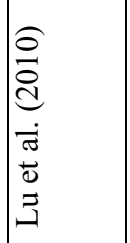 & 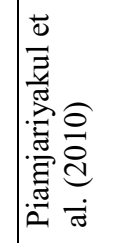 & 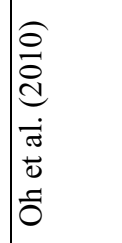 & 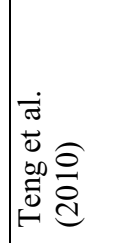 & 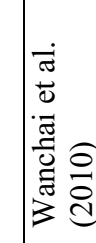 \\
\hline 4 & - & $\cong$ & \pm & $\cong$ & 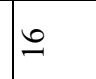 & - & $\cong$ & 7 & $\stackrel{\sim}{~}$ & $\sim$ & $N$ & d & $\sim$ \\
\hline
\end{tabular}


ACCEPTED MANUSCRIPT

\begin{tabular}{|c|c|c|c|c|c|c|c|c|c|c|c|c|c|c|}
\hline 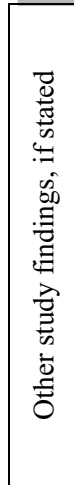 & 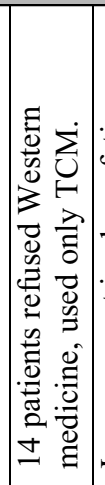 & 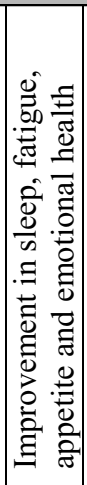 & 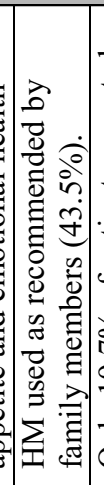 & 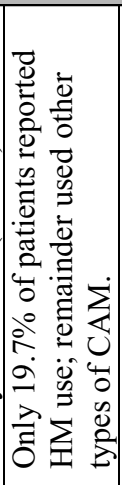 & 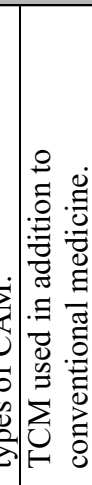 & 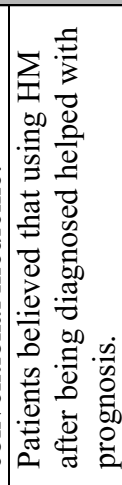 & 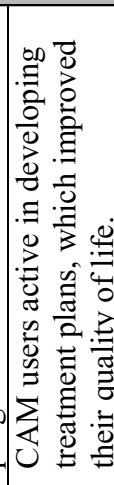 & 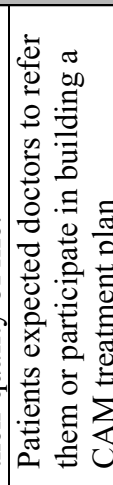 & 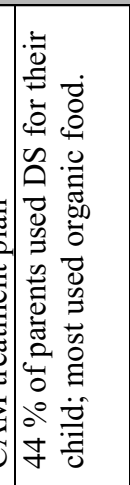 & 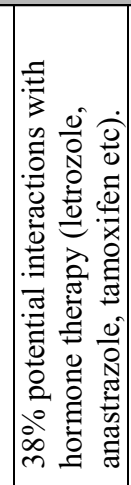 & 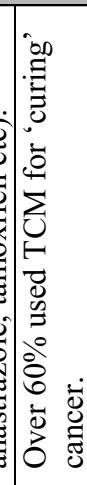 & 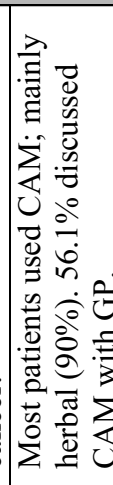 & 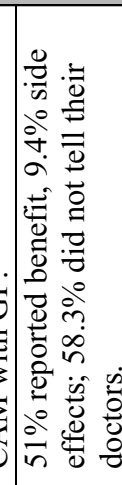 & 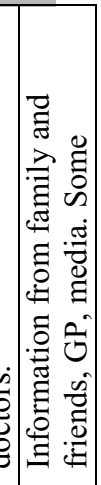 \\
\hline 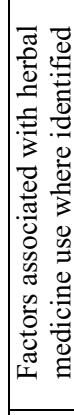 & 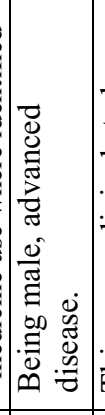 & 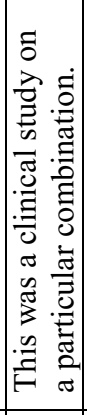 & 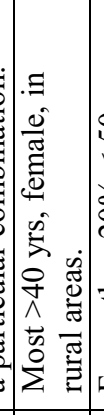 & 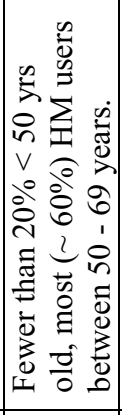 & 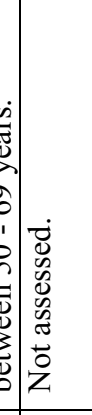 & 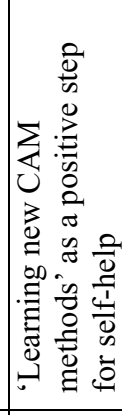 & 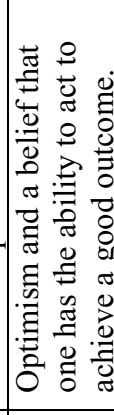 & 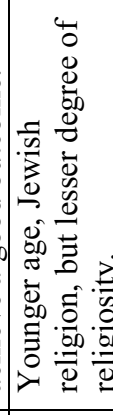 & 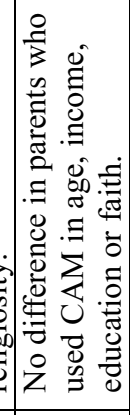 & 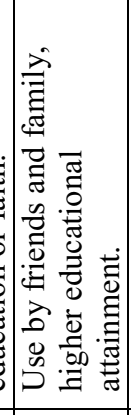 & 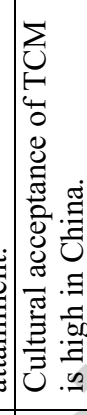 & 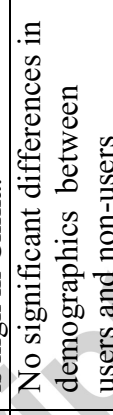 & 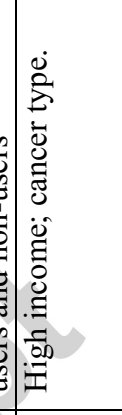 & 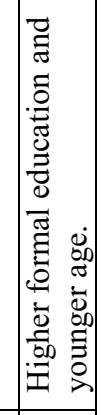 \\
\hline 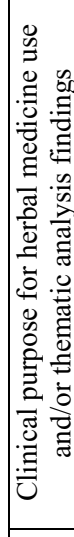 & 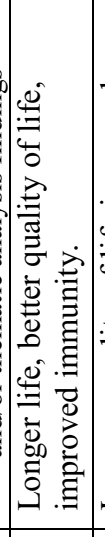 & 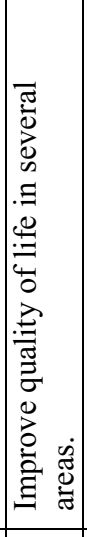 & 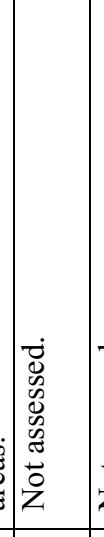 & 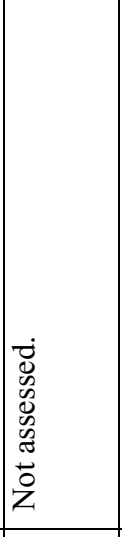 & 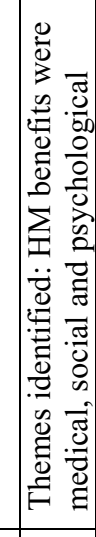 & 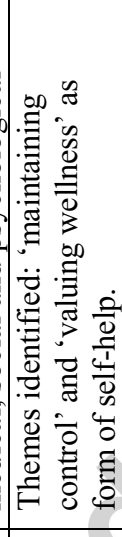 & 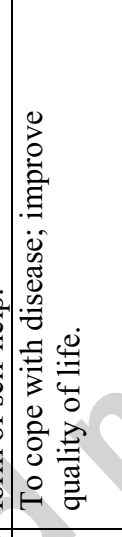 & 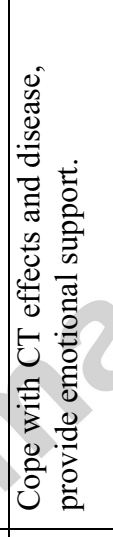 & 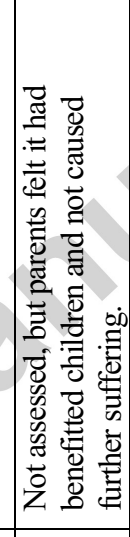 & 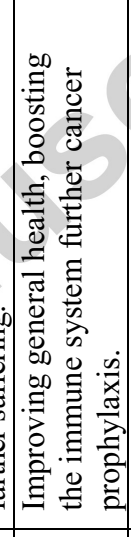 & 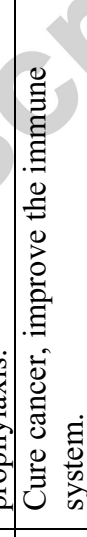 & 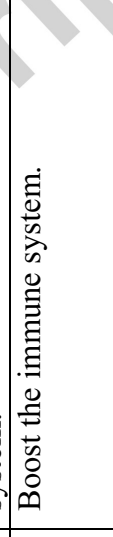 & 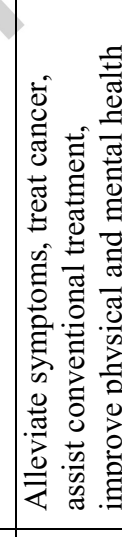 & 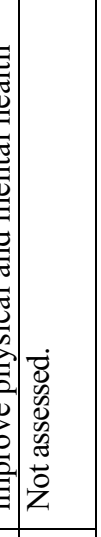 \\
\hline & & 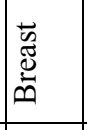 & : & 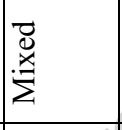 & $\begin{array}{l}\vec{\Xi} \\
\dot{x} \\
\Sigma \\
\end{array}$ & 㤎 & 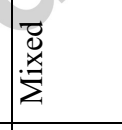 & 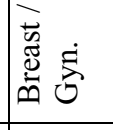 & 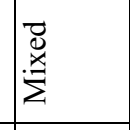 & 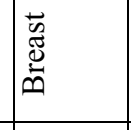 & 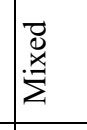 & 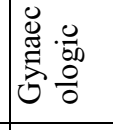 & $\begin{array}{l}\vec{\Xi} \\
\ddot{\ddot{x}} \\
\stackrel{x}{\Sigma}\end{array}$ & ల్ర \\
\hline & & $\infty$ & : & & $a$ & $\simeq$ & $\approx$ & $\stackrel{n}{\sim}$ & 2 & $\tilde{n}$ & लि & 5 & $\stackrel{\infty}{\sim}$ & $\overrightarrow{\bar{N}}$ \\
\hline & & & . & 1 & ' & & $\alpha$ & & . & $\begin{array}{l}\stackrel{\circ}{2} \\
2 \\
2\end{array}$ & $\mid \begin{array}{c}\tilde{N} \\
\infty \\
\infty\end{array}$ & & & $\bar{\infty}$ \\
\hline$\sum_{\bar{\Sigma}}^{\frac{\pi}{\Sigma}}$ & 采 & 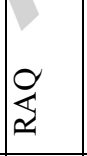 & 临 & 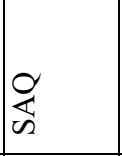 & $\vec{w}$ & $\overline{\tilde{w}}$ & $\vec{w}$ & 隹 & $\frac{g}{d}$ & $\frac{a}{\frac{1}{4}}$ & 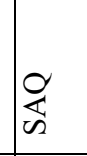 & $\begin{array}{l}0 \\
\frac{8}{4} \\
\text { m }\end{array}$ & s & L \\
\hline 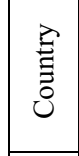 & 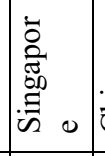 & ठี & 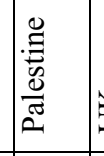 & 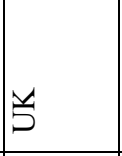 & हू. & 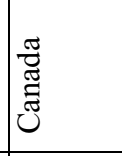 & 死 & 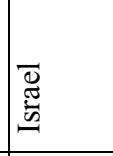 & 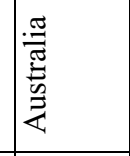 & 5 & 俈 & 童 & 疋 & 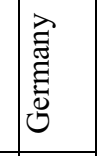 \\
\hline & 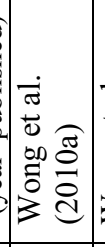 & 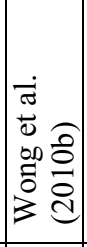 & 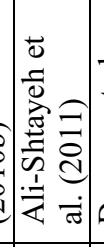 & 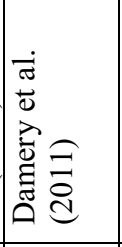 & 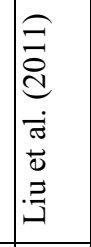 & 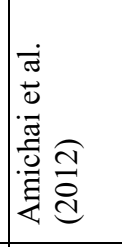 & 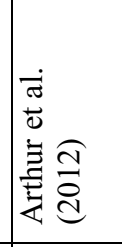 & 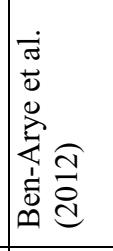 & 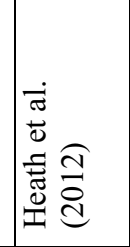 & 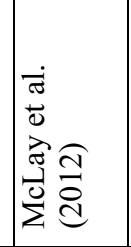 & 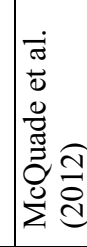 & 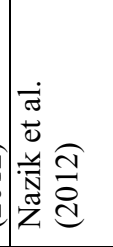 & 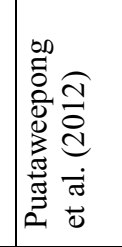 & 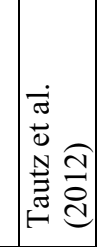 \\
\hline z & 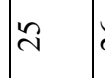 & i & $\hat{\sim}$ & $\stackrel{\infty}{\sim}$ & ते & $m$ & $m$ & $\tilde{m}$ & $\tilde{m}$ & ল & $\approx$ & m & $m$ & $m$ \\
\hline
\end{tabular}




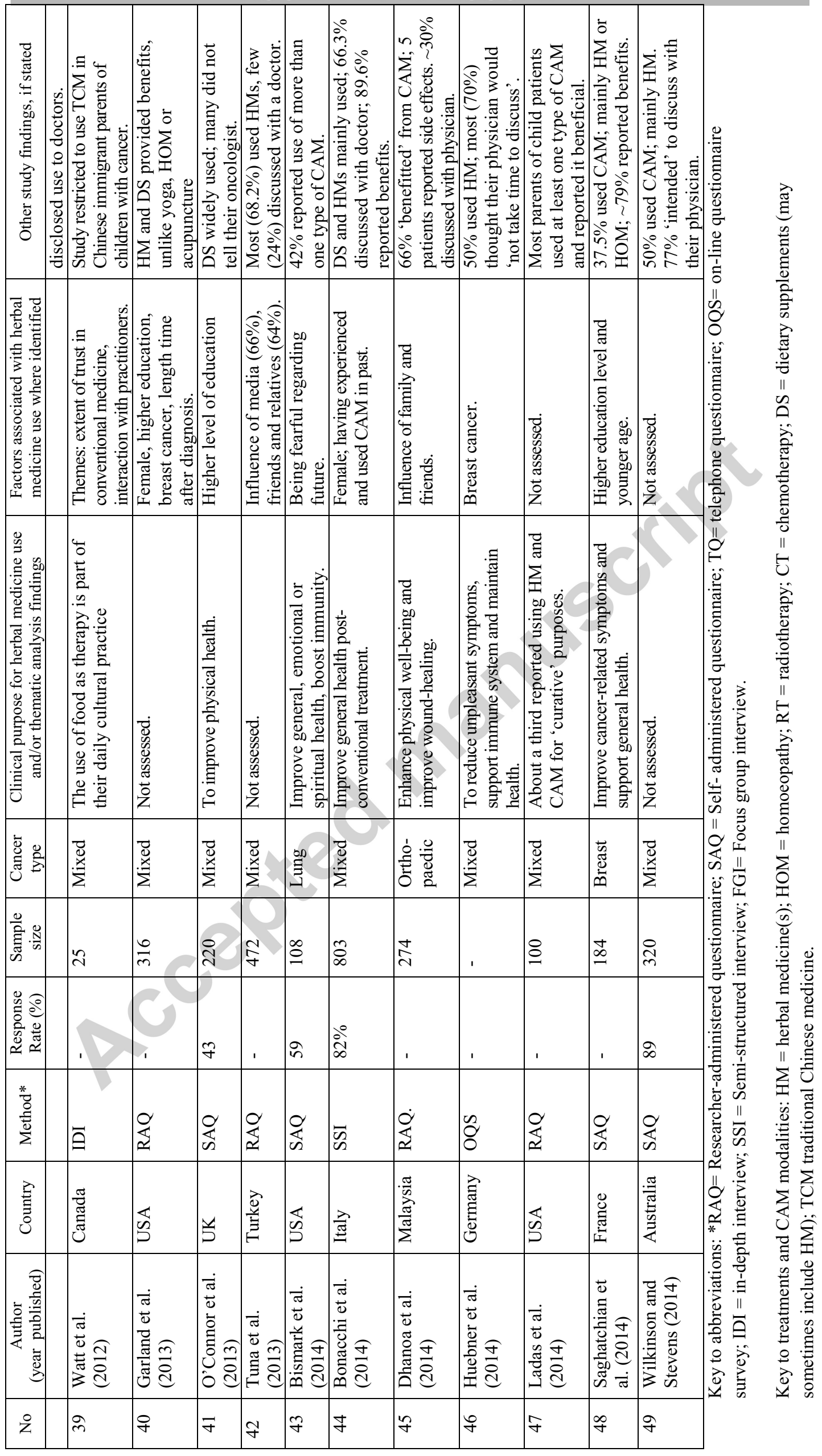


Factors associated with herbal medicines use as part of a CAM regime

In general, the results supported other studies investigating the frequency of general CAM use which have found that a younger age, higher level of education and income, ethnicity and being female, were linked to HM-CAM use, as detailed in table 1. As far as cancer patients are concerned, this diagnosis appears to act as an impetus to using HM-CAM, with the intention of improving general health to 'fight disease' as well as cope with side effects of conventional drug treatment, in a way not usually associated with other disease states.

The more serious disease states were associated with CAM which included HM and dietary supplements (HM-CAM). Multiple chemotherapy treatment was related to higher HM-CAM usage and many patients started using CAM (of any type) only after being diagnosed with cancer. However, those who had used HM-CAM for other purposes were also more likely to be associated with its use in cancer. A greater use of HM-CAM was noted in cancer patients who were in a recurrent or metastatic stage (e.g. Cui et al., 2004) and the longer the time since the initial cancer diagnosis, the more likely patients were to use HMs (Salminen et al., 2004). These reasons may be related to other factors such as 'fearfulness about the future' and 'anxiety about possible recurrence', which were also linked with a greater tendency to use HM-CAM (Correa-Velez et al., 2003, Bismark et al., 2014).

Experiences reported by patients after taking herbal medicines

The recorded incidence of herbal use varies widely, i.e. between $10.8 \%$ and $90.2 \%$, but all the studies reviewed showed that at least $55 \%$ of patients believed they had had benefited, whereas few patients ( $8 \%$ to 18\%) reported negative effects (Damery et al., 2011, Nazik et al., 2012, Molassiotis et al., 2006, Chen et al., 2008, Tuna et al., 2013, Bonacchi et al., 2014).

Perceived beneficial experiences: Previous studies have reported positive effects after HMCAM use but results varied greatly. Between $22 \%$ and $90 \%$ of patients said they had experienced benefits, the most common being relief of pain, dyspepsia and fever, and improved appetite and patterns of sleep (Oh et al., 2010, Molassiotis et al., 2005, Puataweepong et al., 2012, Hyodo et al., 2005, Teng et al., 2010, Ladas et al., 2014, Trevena and Reeder, 2005). Other perceived benefits were a greater ability to cope with the illness and its treatment or specific effects in relieving pain and adverse effects of conventional medicine including chemotherapy (i.e. nausea), as well as alleviating severe depression or anxiety and improving emotional health, as shown in table 1. 
Perceived negative experiences reported by patients after taking HMs: Negative effects from HM-CAM reported by cancer patients ranged from $3 \%$ to $9.4 \%$ and included pain, dyspepsia, abdominal pain and fatigue (Oh et al., 2010, Molassiotis et al., 2005, Puataweepong et al., 2012, Hyodo et al., 2005, Trevena and Reeder, 2005). As with perceived benefit, it is not possible to ascribe all of these to the HM-CAM treatment and some may be due to progression of the disease.

Other findings of the review

Concurrent use with conventional therapies: most studies found that over $50 \%$ of patients who used HM-CAM did so along with conventional medicines (e.g. Nazik et al., 2012, Gupta et al., 2005, Helyer et al., 2006), many to treat specifically the adverse effects of conventional treatment (e.g. Gupta et al., 2005, McLay JS et al., 2012).

Informing medical practitioners of HM use: differences were noted between countries but the number of studies cited was insufficient to draw any conclusions. Most strikingly, two US and two UK studies reported that the majority of patients surveyed did not tell their doctor of their HM-CAM use, whereas in two Australian studies, the majority either informed or intended to inform their doctor. In Turkey, Italy, Israel and Germany (1 study each), most patients discussed their HM-CAM use with their GP, but in Thailand, most did not (1 study).

Discussion

Many studies have investigated the use of CAM generally in cancer patients, which is commonplace (e.g. Teng et al 2010), and in some cases this has included HM (including dietary supplements). HM is the main form of CAM which can interact with conventional drugs, so the implications of HM use are more serious (e.g. Alsanad et al 2014) and therefore formed the focus of this investigation. As most users of CAM use more than one modality, we have examined all studies which specified the HM as part of their regime. Although in traditional Chinese medicine (TCM) for example, combining conventional with herbal medicine in cancer treatment is endorsed by physicians and may be beneficial (e.g. Hu et al 2015; Cui et al 2004), patients usually used HM-CAM on their own initiative and without informing their doctor, and many considered it was not necessary to do so.

The most common reasons for taking HM-CAM found in this study were linked to the desire to improve physical and mental symptoms and quality of life, and to help deal with the disease and its unpleasant treatment. As CAM is not sanctioned officially by most medical 
authorities, and not usually covered by public insurance schemes, it requires independent research into self-care health options, for example by using the internet and media. This may be a reflection of the findings that younger patients and those of a higher educational and financial status were associated with a higher use of HM-CAM.

This study also showed that most of the relevant information on HM-CAM is available in the mainstream, peer-reviewed literature. A comprehensive set of Thai databases compiled from local studies was used as an example for exploration; however, it provided no new information and did not even identify two Thai clinical studies published internationally (Piamjariyakul et al 2010; Puataweepong et al. 2012). This is understandable since authors prefer to publish in SCI journals, although the results cannot be extrapolated elsewhere until further studies have been done.

\section{Conclusions}

This review identified several indicators for cancer patients who are most likely to take HMCAM, using information taken from Medline. Fears that the results of local studies published regionally are being missed, at least in the case of Thailand, appeared to be unfounded. In addition to patient characteristics as described above, the use of HM-CAM was also associated with the type and stage of cancer and the side-effects of conventional treatment experienced. However, interpreting the specific clinical purposes why patients decide to use HM-CAM, and what they expected of and experienced from the treatments, is hampered by a lack of standard terminology and thematic coding. Patients' own descriptions are too variable and overlapping for meaningful comparison, but even so, most the categories relate to a desire to be actively involved in treatment, to improve general health and aid recovery. The impetus to use HM-CAM comes mainly from patients, rather than practitioners, except in China where integration of TCM and conventional medicine for cancer treatment is more common.

\section{References}

Ali-shtayeh, M., Jamous, R. 2011. Herbal preparation use by patients suffering from cancer in Palestine. Complementary Therapies in Clinical Practice, 17, 235-240.

Alsanad S. M., Williamson E. M., Howard R. L. 2014. Cancer patients at risk of herb/food supplement-drug interactions: A systematic review. Phytotherapy Research 28:1749-1755 
Amichai, T., Grossman, M. Richard, M. 2012. Lung cancer patients' beliefs about complementary and alternative medicine in the promotion of their wellness. European Journal of Oncology Nursing, 16, 520-527.

Arthur, K., Belliard, J., Hardin, S., Knecht, K., Chen, C., Montgomer, S. 2012. Practices, attitudes, and beliefs associated with complementary and alternative medicine (CAM) use among cancer patients. Integrative Cancer Therapies, 11, 232-242.

Astin, J., Reilly, C., Perkins, C., Child, W. 2005. Breast cancer patients' perspectives on and use of complementary and alternative medicine: a study by the Susan G. Komen Breast Cancer Foundation. Journal of the Society for Integrative Oncology, 4, 157-169.

Ben-arye, E., Schiff, E., Steiner, M., Keshet, Y., Lavie, O. 2012. Attitudes of patients with gynecological and breast cancer toward integration of complementary medicine in cancer care. International Journal of Gynecological Cancer, 22, 146-153.

Bismark, R., Chen, H., Dy, G., Gage-bouchard, E., Mahoney, M. 2014. Complementary and alternative medicine use among patients with thoracic malignancies. Supportive Care in Cancer, 22, 1857-1866.

Bonacchi, A., Fazzi, L., Toccafondi, A., Cantore, M., Mambrini, A., Muraca, M., Banchelli, G., Panella, M., Focardi, F., Calosi, R. 2014. Use and Perceived Benefits of Complementary Therapies by Cancer Patients Receiving Conventional Treatment in Italy. Journal of Pain and Symptom Management, 47, 26-34.

Chen, Z., Gu, K., Zheng, Y., Zheng, W., Lu, W., Shu, X. 2008. The use of complementary and alternative medicine among Chinese women with breast cancer. The Journal of Alternative and Complementary Medicine, 14, 1049-1055.

Correa-velez. I., Clavarino, A., Barnett, A, Eastwood, H. 2003. Use of complementary and alternative medicine and quality of life: changes at the end of life. Palliative Medicine, 17, 695-703.

Cui, Y., Shu, X., Gao, Y., Wen, W., Ruan, Z., Jin, F., Zheng, W. 2004. Use of complementary and alternative medicine by Chinese women with breast cancer. Breast Cancer Research and Treatment, 85, 263-270.

Damery, S., Gratus, C., Grieve, R., Warmington, S., Jones, J., Routledge, P., Greenfield, S., Dowswell, G., Sherriff, J., Wilson, S. 2011. The use of herbal medicines by people with cancer: a cross-sectional survey. British Journal of Cancer, 104, 927-933.

Dhanoa, A., Yong, T., Yeap, S., Lee, I., Singh, V. 2014. Complementary and alternative medicine use amongst Malaysian orthopaedic oncology patients. BMC Complementary and Alternative Medicine, 14, 404.

Ernst, E. 2009. Complementary and alternative medicine (CAM) and cancer: the kind face of complementary medicine. International Journal of Surgery, 7, 499-500. 
Garland, S., Valentine, D., Desai, K., Li, S., Langer, C., Evans, T., Mao, J. 2013.

Complementary and Alternative Medicine Use and Benefit Finding Among Cancer Patients. The Journal of Alternative and Complementary Medicine, 19, 876-881.

Goey AK, Beijnen JH, Schellens JH (2014). Herb-drug interactions in oncology. Clinical Pharmacology and Therapeutics. 95, 354-355

Gulluoglu, B., Cingi, A., Cakir, T., Barlas, A. 2008. Patients in northwestern Turkey prefer herbs as complementary medicine after breast cancer diagnosis. Breast Care, 3, 269-273.

Gupta, D., Lis, C., Birdsall, T., Grutsch, J. 2005. The use of dietary supplements in a community hospital comprehensive cancer center: implications for conventional cancer care. Supportive Care in Cancer, 13, 912-919.

Hann, D., Baker, F., Denniston, M., Entrekin, N. 2005. Long-term breast cancer survivors' use of complementary therapies: perceived impact on recovery and prevention of recurrence. Integrative Cancer Therapies, 4, 14-20.

Heath, J., Oh, L., Clarke, N., Wolfe, J. 2012. Complementary and alternative medicine use in children with cancer at the end of life. Journal of Palliative Medicine, 15, 1218-1221.

Heinrich M, Edwards S, Moerman DE, Leonti M (2009). Ethnopharmacological field studies: a critical assessment of their conceptual basis and methods. Journal of Ethnopharmacology, $124,1-17$.

Helyer, L., Chin, S., Chui, B., Fitzgerald, B., Verma, S., Rakovitch, E., Dranitsaris, G., Clemons, M. 2006. The use of complementary and alternative medicines among patients with locally advanced breast cancer--a descriptive study. BMC Cancer, 6, 39.

Henderson, J., Donatelle, R. 2003. Complementary and alternative medicine use by women after completion of allopathic treatment for breast cancer. Alternative Therapies in Health and Medicine, 10, 52-57.

$\mathrm{Hu}$ YC, Wu CT, Lai JN, Tsai YT (2015). Detection of a negative correlation between prescription of Chinese herbal products containing coumestrol, genistein or daidzein and risk of subsequent endometrial cancer among tamoxifen-treated female breast cancer survivors in Taiwan between 1998 and 2008: A population-based study. J Ethnopharmacol. 169:356-62

Huebner, J., Muenstedt, K., Prott, F., Stoll, C., Micke, O., Buentzel, J., Muecke, R., Senf, B. 2014. Online Survey of Patients with Breast Cancer on Complementary and Alternative Medicine. Breast Care, 9, 60-63.

Humpel, N., Jones, S. C. 2006. Gaining insight into the what, why and where of complementary and alternative medicine use by cancer patients and survivors. European Journal of Cancer Care, 15, 362-8.

Hyodo, I., Amano, N., Eguchi, K., Narabayashi, M., Imanishi, J., Hirai, M., Nakano, T., Takashima, S. 2005. Nationwide survey on complementary and alternative medicine in cancer patients in Japan. Journal of Clinical Oncology, 23, 2645-2654. 
Kim, S., Kim, K., Park, J., Shin, J., Kim, S., Park, J., Park, E., Seo, H. 2013. Factors Associated with Discontinuation of Complementary and Alternative Medicine among Korean Cancer Patients. Asian Pacific Journal of Cancer Prevention, 14, 225-230.

Klepser, T., Doucette, W., Horton, M., Buys, L., Ernst, M., Ford, J., Hoehns, J., Kautzman, H., Logemann, C., Swegle, J. 2000. Assessment of patients' perceptions and beliefs regarding herbal therapies. Pharmacotherapy: The Journal of Human Pharmacology and Drug Therapy, 20, 83-87.

Kremser, T., Evans, A., Moore, A., Luxford, K., Begbie, S., Bensoussan, A., Marigliani, R., Zorbas, H. 2008. Use of complementary therapies by Australian women with breast cancer. Breast, 17, 387-94.

Ladas, E., Rivas, S., Ndao, D., Damoulakis, D., Bao, Y., Cheng, B., Kelly, K., Antillon, F. 2014. Use of traditional and complementary/alternative medicine (TCAM) in children with cancer in Guatemala. Pediatric Blood and Cancer, 61, 687-692.

Lengacher, C., Bennett, M., Kip, K., Gonzalez, L., Jacobsen, P., Cox, C. 2006. Relief of symptoms, side effects, and psychological distress through use of complementary and alternative medicine in women with breast cancer. Oncology Nursing Forum, 33, 97-104.

Liu, C., Tang, W., Wang, H., Lee, K. 2011. Cancer patients' experience of combined treatment with conventional and traditional Chinese medicine: A biopsychosocial phenomenon. Cancer Nursing, 34, 495-502.

Lu, J., Tsay, S., Sung, S. 2010. Taiwanese adult cancer patients' reports of using complementary therapies. Cancer Nursing, 33, 320-326.

Mao, J., Palmer, S., Desai, K., Li, S., Armstrong, K., Xie, S. 2012. Development and validation of an instrument for measuring attitudes and beliefs about complementary and alternative medicine (CAM) use among cancer patients. Evidence-Based Complementary and Alternative Medicine, 2012:798098..

McLay, J., Stewart, D., George, J., Rore, C., Heys, S. 2012. Complementary and alternative medicines use by Scottish women with breast cancer. What, why and the potential for drug interactions? European Journal of Clinical Pharmacology, 68, 811-819.

McQuade, J., Meng, Z., Chen, Z., Wei, Q., Zhang, Y., Bei, W., Palmer, J., Cohen, L. 2012. Utilization of and attitudes towards traditional Chinese medicine therapies in a Chinese cancer hospital: a survey of patients and physicians. Evidence-Based Complementary and Alternative Medicine, 2012 :504507.

Molassiotis, A., Fernadez-ortega, P., Pud, D., Ozden, G., Scott, J., Panteli, V., Margulies, A., Browall, M., Magri, M. Selvekerova, S. 2005. Use of complementary and alternative medicine in cancer patients: a European survey. Annals of Oncology, 16, 655-663.

Molassiotis, A., Panteli, V., Patiraki, E., Ozden, G., Platin, N., Madsen, E., Browall, M., Fernandez-ortega, P., Pud, D., Margulies, A. 2006. Complementary and alternative medicine use in lung cancer patients in eight European countries. Complementary Therapies in Clinical Practice, 12, 34-39. 
Nazik, E., Nazik, H., Api, M., Kale, A., Aksu, M. 2012. Complementary and alternative medicine use by gynecologic oncology patients in Turkey. Asian Pacific Journal of Cancer Prevention 13, 21-25.

O’Connor, N., Graham, D., O’Meara, A., Devins, M., Jennings, V., O’leary, D., O’Reilly, M. 2013. The use of complementary and alternative medicine by Irish pediatric cancer patients. Journal of Pediatric Hematology/Oncology, 35, 537-542.

Oh, B., Butow, P., Mullan, B., Beale, P., Pavlakis, N., Rosenthal, D., Clarke, S. 2010. The use and perceived benefits resulting from the use of complementary and alternative medicine by cancer patients in Australia. Asia-Pacific Journal of Clinical Oncology, 6, 342-349.

Piamjariyakul, U., Williams, P., Prapakorn, S., Kim, M., Park, L., Rojjanasrirat, W., Williams, A. 2010. Cancer therapy-related symptoms and self-care in Thailand. European Journal of Oncology Nursing, 14, 387-394.

Puataweepong, P., Sutheechet, N., Ratanamongkol, P. 2012. A survey of complementary and alternative medicine use in cancer patients treated with radiotherapy in Thailand. EvidenceBased Complementary and Alternative Medicine, 2012:670408.

Saghatchian, M., Bihan, C., Chenailler, C., Mazouni, C., Dauchy, S., Delaloge, S. 2014. Exploring frontiers: Use of complementary and alternative medicine among patients with early-stage breast cancer. The Breast, 23, 279-285.

Salminen, E., Bishop, M., Poussa, T., Drummond, R., Salminen, S. 2004. Dietary attitudes and changes as well as use of supplements and complementary therapies by Australian and Finnish women following the diagnosis of breast cancer. European Journal of Clinical Nutrition, 58, 137-144.

Tautz, E., Momm, F., Hasenburg, A. \& Guethlin, C. 2012. Use of complementary and alternative medicine in breast cancer patients and their experiences: a cross-sectional study. European Journal of Cancer, 48, 3133-3139.

Teng, L., Jin, K., He, K., Bian, C., Chen, W., Fu, K., Zhu, T., Jin, Z. 2010. Use of complementary and alternative medicine by cancer patients at Zhejiang University Teaching Hospital, Zhuji Hospital, China. African Journal of Traditional, Complementary and Alternative Medicines, 7, 322-330.

Thai Government (2014) http://arcbs.bsru.ac.th/web2009/database/thedb.html (accessed August 2015)

Tovey, P., Broom, A., Chatwin, J., Hafeez, M., Ahmad, S. 2005. Patient assessment of effectiveness and satisfaction with traditional medicine, globalized complementary and alternative medicines, and allopathic medicines for cancer in Pakistan. Integrative Cancer Therapies, 4, 242-8.

Trevena, J., Reeder, A. 2005. Perceptions of New Zealand adults about complementary and alternative therapies for cancer treatment. New Zealand Medical Journal, 118, U1787. 
Tuna, S., Dizdar, O., Calis, M. 2013. The prevalence of usage of herbal medicines among cancer patients. Journal of BUON (Balkan Union of Oncology), 18, 1048-51.

Van der weg, F. \& Streuli, R. A. 2003. Use of alternative medicine by patients with cancer in a rural area of Switzerland. Swiss Medical Weekly, 133, 233-40.

Wanchai, A., Armer, J., Stewart, B. Breast cancer survivors' perspectives of care practices in western and alternative medicine. Oncology nursing forum, 2010. Oncology Nursing Society, 494-500.

Watt, L., Gulati, S., Shaw, N., Sung, L., Dix, D., Poureslami, I., Klassen, A. 2012. Perceptions about complementary and alternative medicine use among Chinese immigrant parents of children with cancer. Supportive Care in Cancer, 20, 253-260.

Wilkinson, J., Stevens, M. 2014. Use of complementary and alternative medical therapies (CAM) by patients attending a regional comprehensive cancer care centre. Journal of Complementary and Integrative Medicine, 11, 139-145.

Williams PD, Piamjariyakul U, Ducey K, Badura J, Boltz KD, Olberding K, Wingate A., Williams A. R. 2006. Cancer treatment, symptom monitoring, and self-care in adults: pilot study. Cancer Nursing, 29, 347-55.

Williamson, E. M., Driver, S. B., Baxter, K. Lee, C. R. 2013. Stockley's Herbal Medicines Interactions : a guide to the interactions of herbal medicines, London, Pharmaceutical Press.

Wong-kim, E., Merighi, J. 2007. Complementary and alternative medicine for pain management in US-and foreign-born Chinese women with breast cancer. Journal of Health Care for the Poor and Underserved, 18, 118-129.

Wong, L., Chan, E., Tay, S., Lee, K., Back, M. 2010a. Complementary and alternative medicine practices among Asian radiotherapy patients. Asia Pacific Journal of Clinical Oncology, 6, 357-63.

Wong, L., Wong, C., Leung, P., Lam, W. 2010b. The efficacy of herbal therapy on quality of life in patients with breast cancer: self-control clinical trial. Patient Preference and Adherence, 4, 223.

Zeller, T., Muesnstedt, K., Stoll, C., Schweder, J., Senf, B., Ruckhaeberle, E., Becker, S., Serve, H., Huebner, J. 2013. Potential interactions of complementary and alternative medicine with cancer therapy in outpatients with gynecological cancer in a comprehensive cancer center. Journal of Cancer Research and Clinical Oncology, 139, 357-365. 

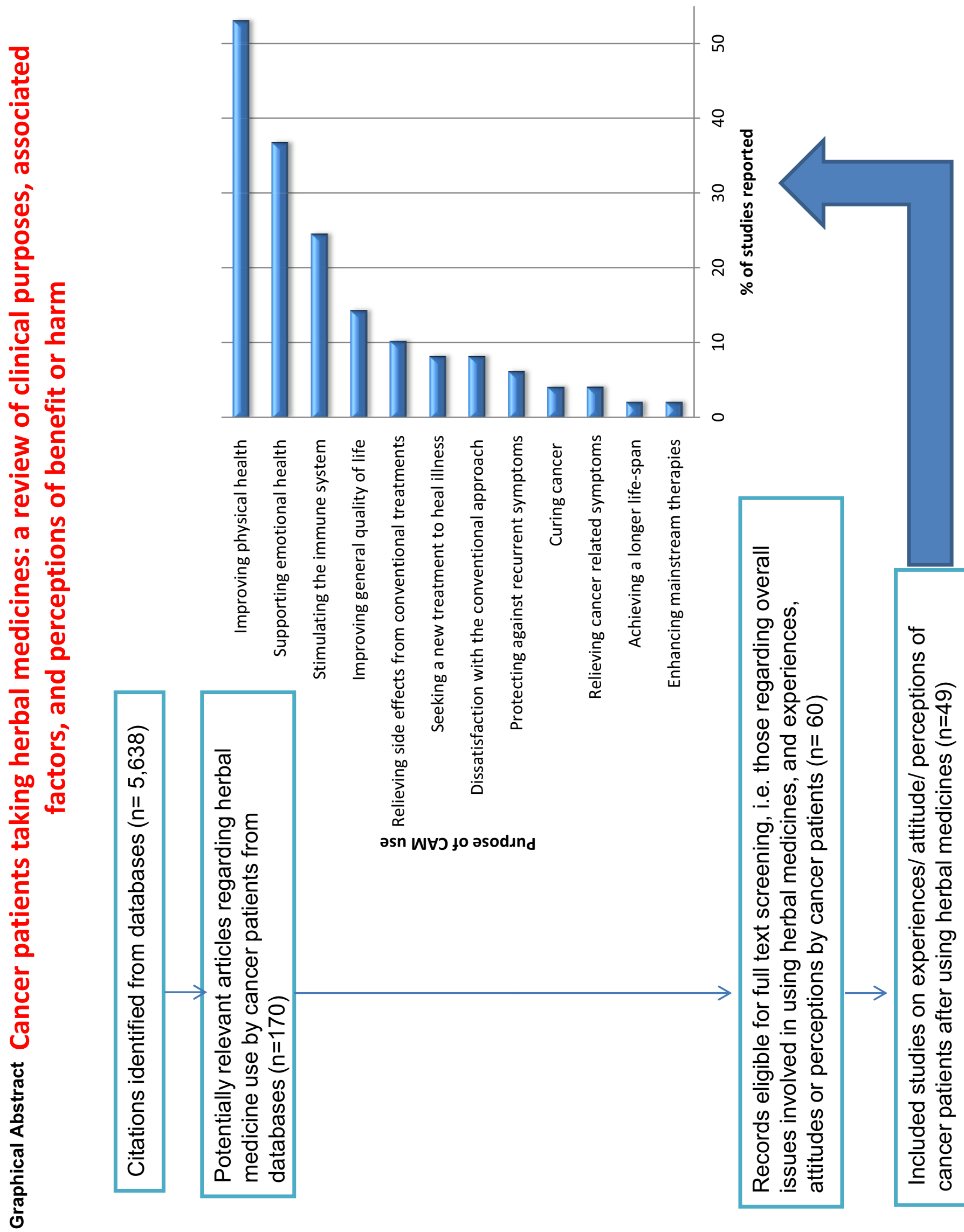